\title{
diffusion-înurdarrentitals
}

The Open-Access Journal for the Basic Principles of Diffusion Theory, Experiment and Application

\section{Polymer dynamics from synthetic to biological macromolecules}

\author{
D. Richter*, R. Biehl*, M. Monkenbusch*, B. Hoffmann**, R. Merkel** \\ *Institut für Festkörperforschung; **Institut für Bio- und Nanosysteme, \\ Forschungszentrum Jülich, D-52425 Jülich, Germany
}

\author{
Corresponding author: \\ Dieter Richter \\ Institut für Festkörperforschung \\ Forschungszentrum Jülich \\ D-52425 Jülich \\ Germany \\ Email: d.richter@fz-juelich.de
}

\begin{abstract}
In soft materials entropic and enthalpic contributions are of similar magnitude and balance each other. Therefore, the macroscopic mechanical and rheological properties and the phase changes are determined to a high degree by thermal motion of the atoms and molecules. Most of the relevant dynamics takes place on mesoscopic length and time scales in between the picosecond atomic scale and the macroscopic frame. Allowing for the proper space time observation window, neutron spin echo (NSE) spectroscopy uniquely allows to address these motions. Here we briefly present some key experimental results on the mesoscopic dynamics of polymer systems, starting from the standard model of polymer motion - the Rouse model. We briefly touch the role of topological confinement as expressed in the reptation model and discuss in some more detail processes limiting the confinement. In the second part we touch on some new developments relating to large scale internal dynamics of proteins by neutron spin echo. We will report results of some pioneering studies which show the feasibility of such experiments on large scale protein motion which will most likely initiate further studies.
\end{abstract}

Keywords: Neutron spin echo, reptation, contour length fluctuation, constraint release, alcohol dehydrogenase, aggregate dynamics, rotational diffusion.

\section{Introduction}

The diffusional motion of long chain flexible polymers constitute fascinating physics and at the same time represent one of the great challenges of modern material sciences. The drive towards the molecular understanding of the complex viscoelastic properties of polymer liquids is the focal point of rheology and connects the classical chemical engineering approach with modern physics [1]. There, the tube model invented by Doi/Edwards [2] and de Gennes [3] has shown itself as the most successful molecular model describing the topological confinement imposed by the mutually interpenetrating 
polymer chains in the melt. In terms of this reptation model a theory of viscoelasticity has been developed that describes the main features of polymer melt rheology.

Large scale protein motions on the other hand are critical for proteins to coordinate precise biological functions. Such dynamics are invoked in genome regulatory proteins, motor proteins, signalling proteins and structural proteins. Structural studies have documented the conformational flexibility in proteins accompanying their activity. Results from macroscopic studies such as biochemical kinetics and single molecule detections have also shown the importance of conformational dynamics and Brownian thermal fluctuations within the proteins or protein complexes. However, the time dependent dynamic processes that facilitate such protein motions remain poorly understood and experimentally nearly untouched [4].

Neutron spin echo spectroscopy is the highest resolution neutron technique and provides time resolution in the 100ns range enabling thereby the access to molecular motion on a mesoscopic time scale between the atomic picosecond scales and the macroscopic times [5]. At that scale the molecular motions of the polymers takes place that underlie their macroscopic viscoelastic behaviour. Similarly the large scale motions of bio molecules occur on similar space time frames. In this contribution we commence with the brief description of the standard model of polymer dynamics, the Rouse model, including its neutron scattering verification [6,7]. Then we address confinement effects and discuss NSE results on the self correlation function of a polymer chain in the melt $[6,8]$. We then turn to the limiting processes of reptation and introduce contour length fluctuation and constraint release phenomena [9] and present some key experimental results [10-12].

Finally, we display some first NSE experiments on the dynamics of alcohol dehydrogenase, a protein which plays a key role in the fermentation processes by yeast. We show that by NSE spectroscopy some first insight into the rotational dynamics as well as some internal motion of the alcohol dehydrogenase tetramer may be achieved [13].

\section{Large scale polymer dynamics}

We start with the simplest "standard model" for the dynamics of a polymer chain in a dense environment the so called Rouse model. This model treats the dynamics of a Gaussian chain in a heat bath. Thereby only entropic forces originating from the conformational chain entropy and random thermal forces are considered. At scales $Q \cdot R_{E}>1$ where $R_{E}$ is the chain end to end distance, in Gaussian approximation the self correlation function for this model assumes a simple form [14]. 


$$
\begin{aligned}
\begin{aligned}
S_{\text {self }}(Q, t)=\exp \left[-\frac{Q^{2}}{6}\left\langle r^{2}(t)\right\rangle\right] \\
=\exp \left[-\frac{Q^{2}}{3}\left(\frac{W \ell^{4}}{\pi} t\right)^{1 / 2}\right]
\end{aligned} \\
\text { i.e. } \quad\left\langle r^{2}(t)\right\rangle=\frac{1}{2}\left(\frac{W \ell^{4}}{\pi} t\right)^{1 / 2}
\end{aligned}
$$

The first line displays the general form of the Gaussian approximation showing that a measurement of the self correlation function yields direct access to the mean square displacement of a diffusing particle. The second line presents the specific prediction of the Rouse model. Thereby, $W=3 \mathrm{k}_{\mathrm{B}} \mathrm{T} /\left(\zeta \ell^{2}\right)$ is the elementary Rouse frequency. It is given by the ratio of the entropic force $3 \mathrm{k}_{\mathrm{B}} \mathrm{T} / \ell^{2}$ and the friction coefficient $\zeta . \ell^{2}$ is the mean square segment length. Note that the Rouse model predicts an anomalous sublinear segment diffusion in time.

Fig. 1 presents a measurement of the segment mean square displacement on a fully protonated melt of polyethylenepropylene of a molecular weight of $M_{w}=80000 \mathrm{~g} / \mathrm{mol}$ [6]. It is evident that the mean square displacement follows very well the predicted $t^{1 / 2}$ behaviour for more than two orders of magnitude in time. Since neutron scattering is a space-time resolving technique, the data do not only provide the time law but also give for each time the magnitude of the mean square displacement. E.g. at a time of $t=10 \mathrm{~ns}$ the segment mean square displacement amounts to $620 \AA^{2}$ or a single segment has travelled about $25 \AA$.

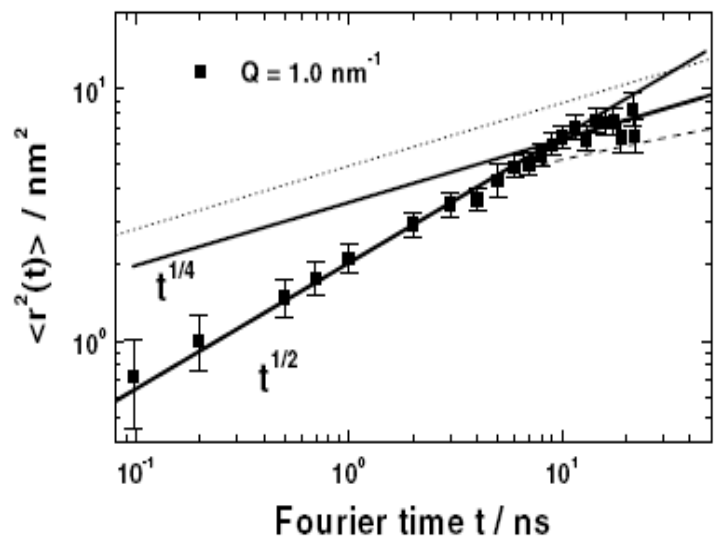

Fig. 1: Time dependent mean square displacement of a PEP segment in the melt at $492 \mathrm{~K}$. The solid line indicates the prediction of the Rouse model [6]. The slowing down at longer times is an indication of a cross over to local repation (see ref [6]). 
For long chains topological chain/chain interactions in terms of entanglements become important and dominate the dynamical behaviour. In the reptation model these constrains are described by a virtual tube which localizes a given chain and limits its motion to a one dimensional Rouse motion inside the tube (local reptation) and a slow diffusive creep motion out of the tube (reptation) [14]. Applying NSE spectroscopy it has become possible to observe the dynamic structure factor $S(Q, t)$ associated with tube confinement and local reptation. de Gennes [3] and Doi/Edwards [2] have formulated tractable analytic expression for the dynamic structure factor. Thereby, they neglected the initial Rouse regime i.e., the derived expression is valid for $t>\tau_{\mathrm{E}}$ once confinement effects become important ( $\tau_{E}$ is the entanglement time, the Rouse relaxation time of an entanglement strand). The dynamic structure factor is composed from two contributions. $S^{\text {loc }}(Q, t)$ and $S^{e s c}(Q, t)$ reflecting local reptation and the escape processes (creep motion) from the tube.

$$
\frac{S_{\text {chain }}(Q, t)}{S_{\text {chain }}(Q)}=\left[1-\exp \left(-\frac{Q^{2} d^{2}}{36}\right)\right] S^{l o c}(Q, t)+\exp \left(-\frac{Q^{2} d^{2}}{36}\right) S^{e s c}(Q, t)
$$

The local reptation part was calculated as [15]

$$
S^{l o c}(Q, t)=\exp \left(\frac{t}{\tau_{0}}\right) \operatorname{erfC}\left[\left(\frac{t}{\tau_{0}}\right)^{1 / 2}\right]
$$

where $\tau_{0}=36 /\left(W \ell^{4} Q^{4}\right)$ and $d$ is the tube diameter. For long chains at short time $S_{\text {chain }}$ decays mainly through local reptation while for longer times the second term describing the creep dominates. The ratio of the two relevant time scales $\tau_{0}$ and $\tau_{\mathrm{d}}$ (disentanglement time) is proportional to $N^{3}$ ( $N$ : number of monomers along the chain). Therefore at intermediate times $\tau_{E}<t<\tau_{d}$ a pronounced plateau in $S_{\text {chain }}(Q, t)$ is predicted. Such a plateau is a signature for a confined motion.

Fig. 2 displays the dynamic structure factor from a long chain polyethylene (PE) melt showing very clearly the tendency to form a plateau at high time [8]. In the spirit of Eq.[2] and neglecting the ongoing decay of $S_{\text {chain }}(Q, t)$ due to local reptation, from the plateau heights we may obtain a first estimate for the size of the confinement. Identifying the plateau levels with a Debye-Waller factor, a confinement length of $d=45 \AA$ is obtained while a full evaluation results in a tube diameter of $d=48 \AA$. We note that at present the de Gennes dynamic structure factor is the only analytical model so far published which is able to describe the dynamic structure factor data.

It is well known that a number of salient properties of entangled polymer melts are only qualitatively in agreement with reptation, indicating the existence of additional processes that release topological confinement [9]. These processes comprise fluctuating chain ends 
which open a route to escape the tube confinement (contour length fluctuations, CLF) and the lateral tube opening by diffusion processes of confining chains (constraint release, CR). While CLF is an effect of the confined chain itself, CR stems from the movements of the chains building the tube which of course undergo the same dynamical processes as the confined chain. Both mechanisms are depicted schematically in Fig. 3.
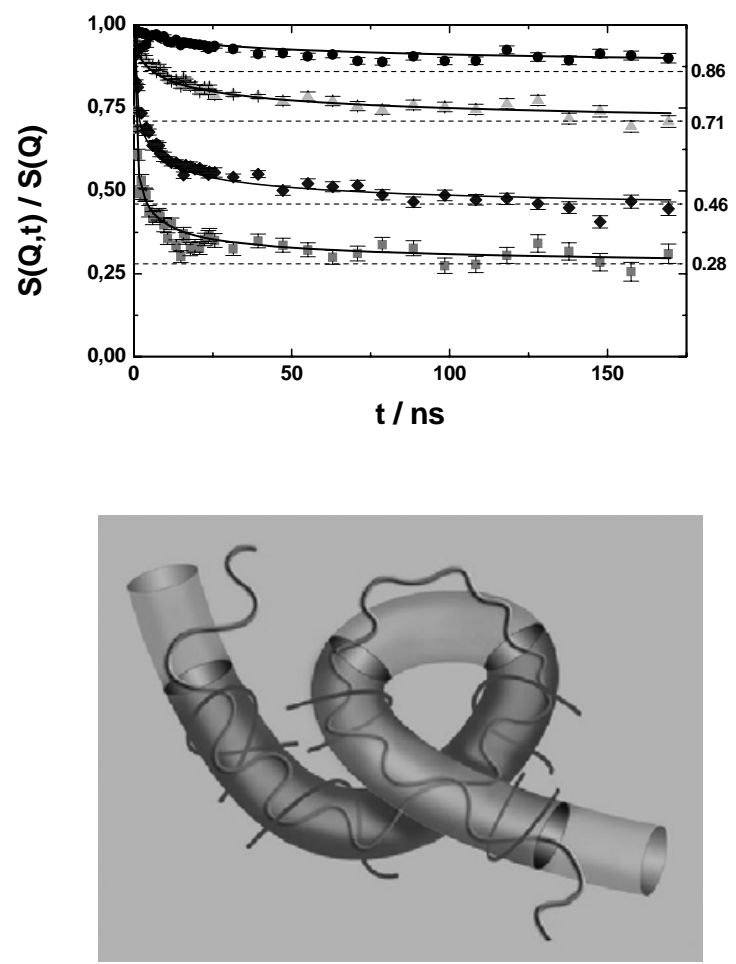

Fig. 2: The dynamic structure factor from a $M_{w}=36 \mathrm{~kg} \mathrm{~mol}^{-1} \mathrm{PE}$ melt at $509 \mathrm{~K}$ as a function of time. The solid lines are a fit with the reptation model (Eq.[2]). The $Q$ values are from the top $Q=0.5$, $0.77,1.15$ and $1.45 \mathrm{~nm}^{-1}$. The horizontal dashed lines display the prediction of the Debye-Waller factor estimate for the confinement size (see text).

Fig. 3: Schematic presentation of the CLF and CR mechanisms: chain end fluctuations lead to a shortening of the effective tube length, while the dissolving of entanglements allow chain motions beyond the initial tube constraints [12].

CLF originate from the fluctuations of the primitive path length of the tube and are a key mechanism for the relaxation at earlier times and are also the basis for hierarchical relaxation processes of branched polymers where CLF are considered to be the fundamental process facilitating the release of side branches.

The CLF effect evolves from the participation of the chain ends in the local reptation process (Fig. 3). Any chain retraction and subsequent expansion leads to a loss of memory of the original confining tube. Thus, effectively the tube becomes shorter with time. Mathematically the problem may be treated as a first passage problem. Whenever a tube contour is visited by the free end it ceases to exist. The functional form of the tube survival probability $\mu(t)$ was derived from scaling arguments [16]. 


$$
\mu(t)=1-\frac{C_{\mu}}{Z}\left(\frac{t}{\tau_{e}}\right)^{1 / 4}
$$

The constant $C_{\mu}=1.5 \pm 0.02$ was obtained from stochastic simulations. $Z=N / N_{e}$ is the number of entanglements and $N_{e}$ the number of segments forming an entanglement strand. Eq.[4] describes quantitatively which chain fraction at a time $t$ is still confined. All parameters are known from the NSE experiments on the dynamics of asymptotically long chains (see above) where the CLF effect does not play a role.

With this knowledge Eq.[4] allows the design of an experiment where the CLF effect may be directly demonstrated [11]. In this experiment the dynamic structure factor of a fully labelled chain is compared with that of an identical chain where the contrast of those segments which are effected by CLF within the experimental time frame was matched. The first case is realized by performing an experiment on a fully protonated chain in a deuterated matrix. In this case the full chain dynamics including the CLF are observed. The second case is realized in a chain where the inner part is protonated while the two outer chain sections of a length which would be effected by CLF are deuterated and thus not visible in the deuterated matrix. In such a case the dynamics should be equal to those of an asymptotically long fully confined chain.

With the known parameters for PE, Eq.[4] gives that at 509K, the experimental temperature, on average on each side 220 monomers are released during an observation time of 190 ns. The above described experiments were performed on two different PE chains of a molecular weight of $25 \mathrm{~kg} \mathrm{~mol}^{-1}$ one of which was fully hydrogenated and the other having deuterated labels of $M_{w}=4 \mathrm{~kg} \mathrm{~mol}^{-1}$ corresponding to 260 monomers on each end. Both were studied in a deuterated matrix of the same molecular weight.

Fig. 4 presents the normalized dynamic structure factor $S(Q, t) / S(Q)$ for different $Q$ values for the two chains. In all cases at short times the structure factor displays a strong initial decay which is due to the initial free Rouse motion. For longer times the decay is strongly reduced, transgressing into the confinement related plateau behaviour. Comparing the levels of decay, we realize that the structure factor from the fully labelled chain decays significantly stronger than that from the corresponding center labelled counter part. Apparently the constraints for the center labelled chain are stronger than those for the chain where the ends are visible. We further note that in the case where the ends were masked the center part of the chain shows exactly the same structure factor as that from a very long chain, signifying directly the action of CLF at the chain ends and the remaining full confinement of the center. The agreement of these two sets of data means that for a $25 \mathrm{~kg} \mathrm{~mol}^{-1}$ chain the effect of constraint release is indeed negligible.

The solid lines in Fig. 4 are a description of the dynamic structure factor in terms of Eq.[2] yielding the same parameters as for asymptotically long chains. In order the depict the CLF mechanism in the dynamic structure factor the escape term in Eq.[2] needs to be modified. The effective tube shortens with time. The time dependent fraction of escaped 
monomers may be incorporated into the structure factor following an approach of Clark and McLeish [17]. Assuming that after time $t$ all monomers from both ends up to a contour length $s(t)=(1-\mu(t)) / 2$ have been released from the tube without introducing any new parameter, a simple analytical expression for the escape term is derived.

$$
S_{C L F}^{e s c}(Q, t)=A\left(2+2 \gamma+e^{-2 \gamma}-4 \gamma s(t)-4 e^{-\gamma s(t)}+e^{-4 \gamma S(t)}\right)
$$

With $A$ a normalization constant and $\gamma=Q^{2} N \ell^{2} / 12$, Eq.[5] replaces $S^{e s c}$ in the original Eq.[2]. This approach is valid as long as $t<\tau_{R}$, where the Rouse time $\tau_{R}$ for a $25 \mathrm{~kg} / \mathrm{mol}$ chain is in the order of 1300ns far beyond the experimental window. The dashed lines in Fig. 4 presents the prediction of Eq.[5] which perfectly describes the data from the fully labelled chain without any further parameter.

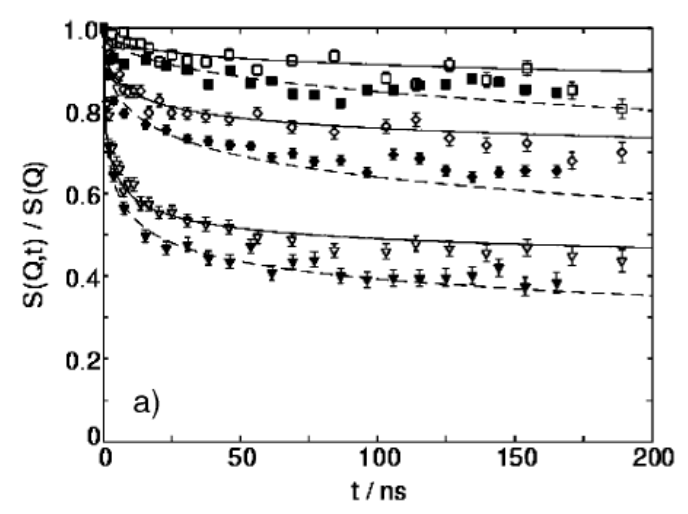

Fig. 4: Dynamic structure factor of a center labelled $25 \mathrm{~kg} \mathrm{~mol}^{-1}$ $\mathrm{PE}$ chain (filled symbols) compared to a fully labelled chain (open symbols) of the same overall molecular weight. $Q$ values (in $\mathrm{nm}^{-1}$ ): 0.5 (squares), 0.77 (diamonds), 0.96 (circles), 1.15 (triangles). Lines: for center labelled chain, pure reptation model (Eq.[2]); for fully labelled $25 \mathrm{~kg} \mathrm{~mol}^{-1}$ chain (dashed) CLF was considered (Eq.[5]).

We now discuss the constraint release process. In order to separate CR effects from the CLF process, one has to consider a labelled chain which is long enough such that end effects like CLF do not play a role $\left(M_{w}=36 \mathrm{~kg} \mathrm{~mol}^{-1}\right)$. The CR effect then may be studied in changing the molecular weight of the matrix chains. Comparing the dynamic structure factor of the long chain in successively shorter matrix chains a clear transition from confined reptation like motion in a long chain matrix to free Rouse motion in a short matrix is observed. This may be best demonstrated in a so called Rouse scaling representation, where the dynamic structure factor is plotted vs. $\left(Q^{2} W \ell^{4} t\right)^{1 / 2}$. In this representation all $S(Q, t)$ data collapse to a single master curve, if Rouse dynamic prevails. On the other hand if topological constraints are evident a length scale comes into play (the tube diameter in the reptation model) and such a scaled dynamic structure factor does not obey a common $Q^{2} t$ scaling.

For a $36 \mathrm{~kg} \mathrm{~mol}^{-1}$ chain neither CLF nor CR play a significant role in the accessible NSE time range and the chains remain confined inside their tube. With decreasing matrix chain 
length (12 or $6 \mathrm{~kg} \mathrm{~mol}^{-1}$, Fig. 5) an increasing loss of confinement is evident in the form of a stronger decay of the dynamic structure factor. This additional relaxation reflects the phenomenon of constraint release: the loosening of the tube confinement due to the motion of the surrounding chains.

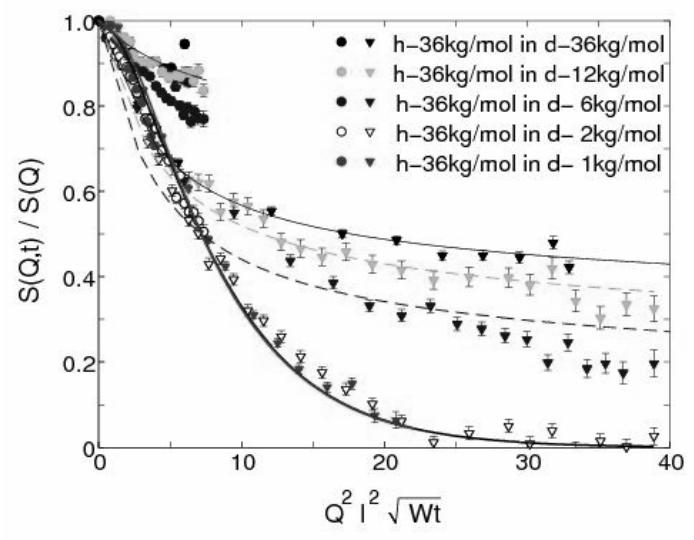

Fig. 5: Dynamic structure factor of a long labelled chain $\left(M_{w}=36 \mathrm{~kg} \mathrm{~mol}^{-1}\right)$ in different shorter matrix chains $\left(M_{w}=36\right.$, 12, $6, \quad 2$ and $1 \mathrm{~kg} \mathrm{~mol}^{-1}$ as indicated in the plot) in a Rouse scaling representation for two different $Q$ values (circles $0.5 \mathrm{~nm}^{-}$ ${ }^{1}$, triangles $\left.1.15 \mathrm{~nm}^{-1}\right)$. The lines correspond to fits with Eq.[2] (reptation) or the Rouse model [14] respectively.

Eventually for a short chain matrix of only about one entanglement length $(2 \mathrm{~kg} / \mathrm{mol})$ the long labelled chain displays the characteristic Rouse scaling. All data collapse on a single master curve: obviously the matrix chains are too short to confine the long chain.

The key question now is which dynamical processes underlie the CR process and lead to the gradual loss of confinement with decreasing matrix chain length. The reptation time of the matrix chain [e.g. $\tau_{d}(12 \mathrm{~kg} / \mathrm{mol}) \cong 5000 \mathrm{~ns}$ ] is far beyond the experimental time range. But nevertheless for the $12 \mathrm{~kg} \mathrm{~mol}^{-1}$ matrix which is well entangled, the effect of CR is significant. This fact demonstrates that for an estimation of the characteristic time scale all dynamical processes which are known to determine segmental motion have to be taken into account. These processes include next to the reptational creep also CLF of the chain ends.

In order to separate the different dynamical processes the following experiment has been designed: for a long chain in a short matrix CLF is negligible. Therefore the effect of CR resulting from the matrix chains may be observed. On the other hand the dynamics of a short chain in a long matrix is dominated by CLF processes - no constraint release of the matrix chains can occur in the time scale of observation. Comparing the dynamic structure factors of such a corresponding pairs of samples the contribution from CLF and $\mathrm{CR}$ of the shorter chain may be separated.

Fig. 6 shows the result for a $12 \mathrm{~kg} / \mathrm{mol}$ chain in a $36 \mathrm{~kg} / \mathrm{mol}$ matrix and vice versa. The long chain relaxation by a constraint release in the short matrix is obviously identical to the relaxation of the confined short chains by contour length fluctuations. I.e. in the 
$12 \mathrm{~kg} / \mathrm{mol}$ matrix the CR visible in the long chain dynamics may be traced to the CLF of the matrix chains. This experiment shows that CLF alone can cause a constraint release effect. This phenomenon has not yet been considered in rheological theories for chain relaxation and needs to be included.

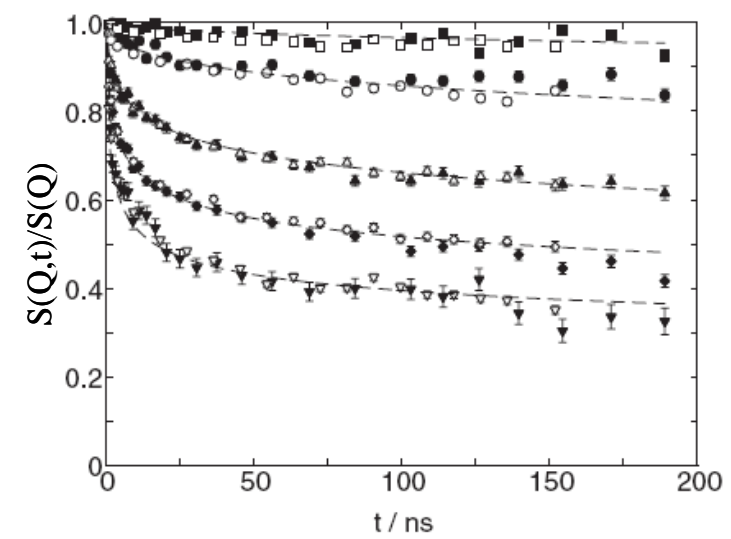

Fig. 6: Dynamic structure factor of a $36 \mathrm{~kg} / \mathrm{mol}$ chain in a $12 \mathrm{~kg} / \mathrm{mol}$ matrix (solid symbols) and vice versa (open symbols). $Q$ values (in $\mathrm{nm}^{-1}$ ): squares 0.3 , circles 0.5 , uppointing triangles 0.77 diamonds 0.96, and downpointing triangles 1.15. Lines are just guides for the eye.

Concluding this paragraph we may emphasize that neutron spin echo spectroscopy in combination with synthetic chemistry is able to unravel the important dynamical mechanisms ruling the complex chain dynamics in polymer melts on a molecular scale. We have demonstrated the astonishing validity of the Rouse model in its space time frame. We then have shown the molecular existence of the tube confinement in long chain polymer melts, thereafter we have eluded to mechanisms inherent to the tube model which limit the confinement. Both - the contour length fluctuations as well as constraint release mechanisms - have been shown on a molecular level. Using sophisticated anionic polymerization chemistry the CLF mechanism which dismantles the tube from its end was unambiguously demonstrated laying also the molecular foundation for the basic process in the hierarchical relaxation of branched polymers. Finally, with regards to constraint release processes the NSE results show that constraint release processes may already occur at earlier times through the CLF mechanism of the confining matrix.

\section{Large scale dynamics in biopolymers}

Now, with the study of polymer dynamics reaching some maturity, the next challenge will be to unravel the large scale motion of biopolymers and to find out to what extent these dynamics play a role in their function. While at present the conformational dynamics on local scales have been successfully approached by e.g. time dependent crystallography [18], large scale dynamics such as protein domain motions remain basically untouched experimentally, because of the lack of techniques to study these large scale correlated motions. A first successful NSE approach on the dynamics of thermus acquaticus polymerase (Taq) has been reported recently by us in a collaboration with an American group [4]. Here we concentrate on another biopolymer alcohol dehydrogenase 
$(\mathrm{ADH})$ which other than Taq polymerase in solution forms a tetrameric aggregate. The alcohol dehydrogenases are enzymes that are present in many organisms, allowing the interconversion between alcohols and ketones. In humans it catalyzes the oxidation of ethanol and is always present in a form of a dimer allowing thereby the consumption of alcoholic beverages. In yeast it is at the basis of the fermentation process and converts acetaldehyde into ethanol. In the process the cofactor NAD is needed assisting the oxidation reaction.

Fig. 7 displays a schematic structure of a dimer based on crystallographic data. The figure displays the dimer with the two monomeric units clearly visible. Each monomer is build from two domains with a small opening in between where the cofactor NAD is placed which assists the oxidation process at the Zinc active site. One of the questions to answer is to what extent the cofactor may modify the domain dynamics.

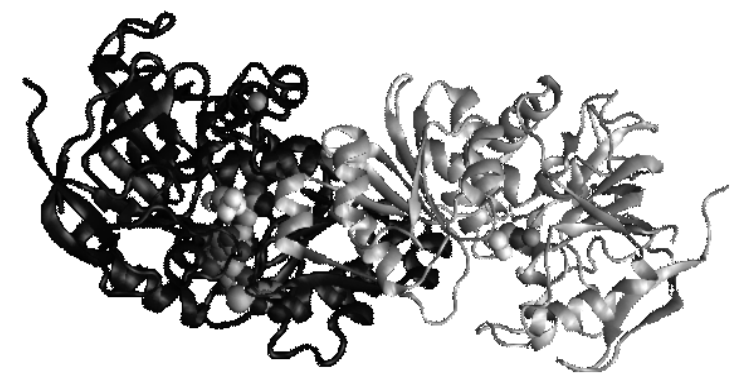

Fig. $\quad$ 7: Dimer of Alcoholdehydrogenase. The molecule presented by spherical caps is the NAD cofactor used in the chemical reaction.

In our studies we used ADH from yeast which forms a tetramer structure. The crystallographic data suggest a crossed arrangement of the two dimers. In order to verify whether in solution a similar tetrameric aggregate is present neutron small angle scattering experiments were performed as a function of concentration. Figure 8 presents the SANS data together with a comparison with a number of models for solution structures. Let us commence with the insert: here for different concentrations the low $Q$ data are presented and there the increasing influence of the structure factor on the SANS data is visible. In the main figure the low concentration data are presented over the full $Q$ range and are compared with different structural models. The solid line reflects the crystal structure which appears to be in perfect agreement with the solution data. Testing the sensitivity of the approach we compare with a situation, where we bring the two dimers in a planar configuration into close contact. The results are presented by the dashed line which in particular at high momentum transfers leads to an underestimation of the scattering data. Furthermore using the programme DAMMIN [19] which builds SANS scattering curves from an arrangement of small mesoscopic spheres also allows a perfect fit of the experimental data (result superimposes with the crystal results) and confirms the crystal based perpendicular arrangement of the two planar structures. Thus, 
we conclude that as in the crystal the ADH tetramer in solution is present in a crossed dimer configuration.
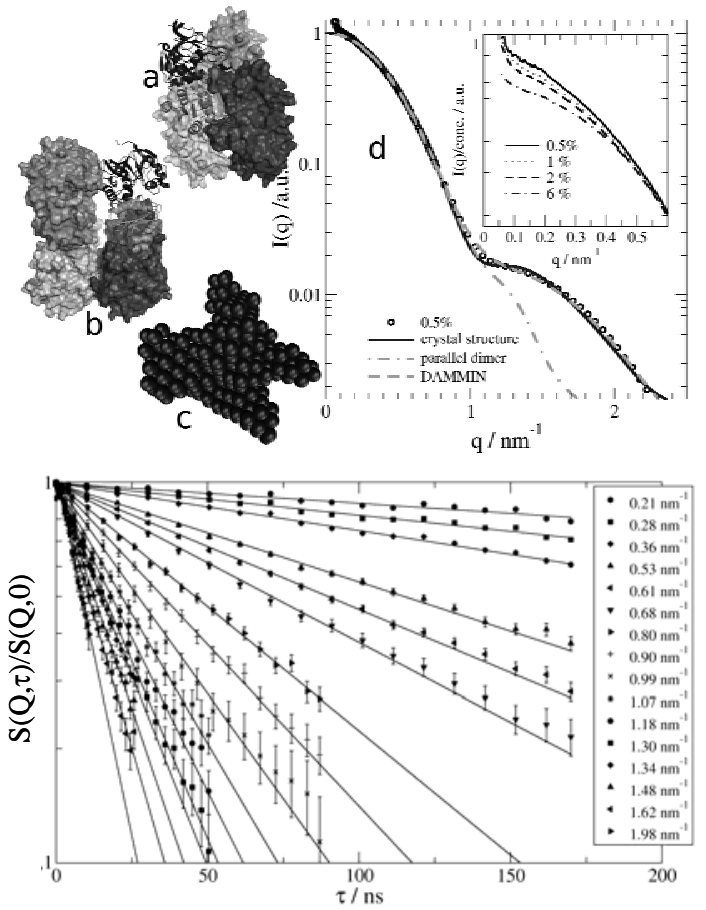

Fig. 8. SANS results on ADH solutions of different concentration (d). Insert: concentration dependent results. The different curves in the main figure display modelling results for various dimer arrangements a) solid line: crystal structure; b) thin solid line: DAMMIN result c) dashed dotted line: planar dimers at close contact.

. Fig 9. Neutron spin echo results on a $1 \% \mathrm{ADH}$ resolution at $5^{\circ} \mathrm{C}$ without cofactor for various momentum transfers

The overall molecular diffusion was measured by dynamic light scattering and was found to be independent of concentration. The translational diffusion coefficient amounts to $D_{D L S}=(2.35 \pm 0.2) 10^{-2} \mathrm{~nm}^{2} / \mathrm{ns}$ at $5^{\circ} \mathrm{C}$ corresponding to a hydrodynamic radius of $R_{H} \cong 4.5 \mathrm{~nm}$.

Neutron spin echo data were measured at different concentrations with and without the cofactor NAD. Figure 9 displays NSE results in a logarithmic fashion for a large number of different momentum transfers $Q$. In each case single exponential fits are included showing that the measured structure factor with great accuracy may be described in terms of a single exponential decay.

Comparing the covered $Q$ range with the SANS data, one realizes that both the range of the structure factor, where intermolecular interactions are important as well as the regime of internal structure are covered. Considering that all data may be described in terms of a single exponential decay, we approximate the spectra in terms of a first cumulant expansion as 


$$
\ln \frac{S(Q, t)}{S(Q, 0)}=-\Gamma(Q) t+\frac{1}{2} K_{2} t^{2} \ldots
$$

where the decay rate of the dynamic structure factor is

$$
\Gamma(Q)=-\lim _{t \rightarrow 0} \frac{\partial}{\partial t} \ln [S(Q, t)]
$$

This gives an effective diffusion coefficient

$$
D_{\text {eff }}(Q)=\frac{\Gamma(Q)}{Q^{2}}
$$

Figure 10a presents the thus obtained effective diffusion coefficients as the function of $Q$ for the different concentrations with and without NAD. The line at low $Q$ indicates the level of the light scattering result.

The experimental data show a strong $Q$ modulation exhibiting a maximum around $Q=1 \mathrm{~nm}^{-1}$. There is significant concentration dependence, though at low $Q$ the data are in agreement with the concentration independent light scattering results. We also see that beyond the statistical error in the low $Q$ flank of the $5 \%$ data the relaxation without NAD is somewhat faster than that including the cofactor. Thus, in the dynamics of $\mathrm{ADH}$ on the scale of the molecule itself we observe significant contributions beyond translational diffusion.

First, we concentrate on the low $Q$ data which are affected by the interactions between the molecules. There the effective diffusion coefficient relates to the diffusion coefficient $\mathrm{D}_{0}$ at infinite dilution by

$$
D_{\text {eff }}(Q)=D_{0} \frac{H(Q)}{S(Q)}
$$

where $H(Q)$ is the hydrodynamic factor and $S(Q)$ is the structure factor. With the measured structure factor the data may immediately be corrected for $S(Q)$. This correction removes the low $Q$ increase of the $5 \%$ data but leaves the results at higher $Q$ untouched. The hydrodynamic factor cannot be measured directly. A first approximation in terms of a Percus Yevic model shows that (i) at a $1 \%$ level the correction factor $H(Q) / S(Q)$ leaves the experimental data practically untouched and (ii) at $5 \%$ the correction is somewhat weaker than the experimentally observed effect. Beyond $Q=0.7 \mathrm{~nm}^{-1}$ the ratio of $H(Q) / S(Q)$ remains constant. Thus, the observed higher $Q$ structure is entirely determined by intra aggregate effects. 


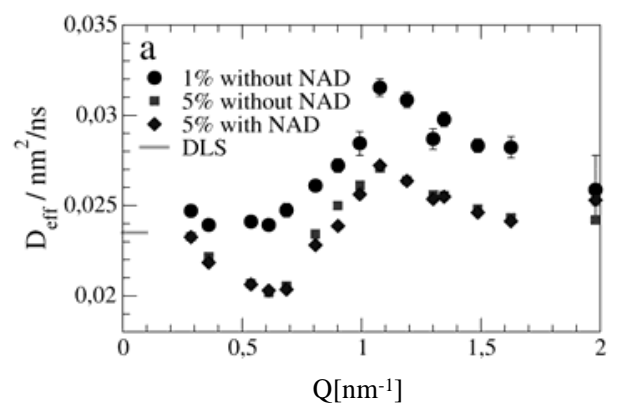

Fig 10. (a) Effective diffusion coefficient $D_{\text {eff }}(Q)$ for 3 different solutions of $\mathrm{ADH}$,

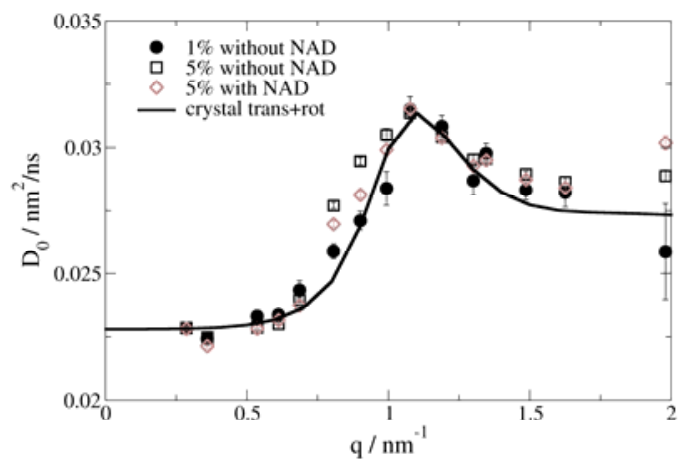

(b) comparison of $D_{\text {eff }}(Q)$ corrected for $S(Q)$ with different models (see text).

The prime reason for a $Q$ dependent structure in $D_{\text {eff }}(Q)$ are rotational motions of the molecule. In a first cumulant approximation the effective diffusion coefficient of a rigid body undergoing translational and rotational diffusion has the following form [20]

$$
\begin{aligned}
D_{\text {eff }}(Q)= & \frac{k_{B} T}{Q^{2}} \times \\
& \left.\frac{\sum_{j K}\left\langle b_{j} e^{i \underline{Q} \underline{r}_{j}}\left(\begin{array}{c}
\underline{Q} \\
Q \times r_{j}
\end{array}\right) \overleftrightarrow{H}\left(\begin{array}{c}
\underline{Q} \\
\underline{Q} \times \underline{r}_{K}
\end{array}\right) b_{K} e^{-i \underline{Q} \underline{r}_{K}}\right\rangle}{\sum_{j K}\left\langle b_{j} e^{i \underline{Q} \underline{r}_{j}}\right.} b_{K} e^{-i \underline{Q} \underline{r}_{K}}\right\rangle
\end{aligned}
$$

Here $\underline{r}_{i}$ and $\underline{r}_{K}$ are the atomic coordinates, $b_{i}$ and $b_{K}$ the corresponding neutron scattering lengths and $\vec{H}$ the mobility tensor. The sums run over all atoms of the molecule or molecular aggregate and the pointed brackets indicate an ensemble average. The denominator resembles the aggregate form factor. The mobility matrix $\vec{H}$ is a $6 x 6$ tensor involving translational and rotational parts including a translational rotational coupling. The further evaluation of Eq.[7] was performed using the program HYDROPRO created by Garcia de la Torre and coworkers [21]. The rotational averaging 
was performed numerically with small step sizes. The calculations with HYDROPRO need as an input the crystallographic coordinates of all atoms.

In Figure $10 \mathrm{~b}$ all data sets for $D_{\text {eff }}(Q)$ at $1 \%, 5 \%$, with and without NAD are compared. Thereby $D_{\text {eff }}(Q)$ was rescaled with the structure factor. We realize that after rescaling the diffusion coefficient in the region above $Q=0.6 \mathrm{~nm}^{-1}$ all data sets are consistent. We note that the data at $5 \%$ solution are of significantly higher statistical accuracy underlining the deviation from pure rotational motion even more strongly. Furthermore the sample with cofactor compared to that without NAD exhibits a somewhat slower dynamics in the low $Q$ flank of the peak in $D_{\text {eff }}(Q)$. Given the overall consistency of the data points and the size of the error bars this difference in the dynamic response appears to be significant and indicates some influence of the binding of NAD on the overall molecular dynamics

Using the crystal based crossed dimer model and normalizing the data to the translational diffusion coefficient obtained by light scattering we obtain the dashed curve in Figure 10b describing the overall shape of $D_{\text {eff }}(Q)$ quite well but misses the proper low and high Q-flanks. These deviations may indicate some additional dynamics which is not depicted by the rigid body rotation. At present we evaluate these differences in terms of a normal mode analysis indicating that they result from fluctuations of the outer more flexible part of the tetramer [13]. In such a picture the differences in the low Q flank of the data from the aggregate with and without the cofactor seem to indicate a significant difference of the configurational stiffness of the protein depending on the cofactor presence.

\section{Conclusion and outlook}

We have presented some representative results from neutron spin echo spectroscopy on the dynamics of macromolecules. In the case of linear chains we have shown that the large scale dynamics may be well understood in terms of confinements giving rise to tube constraints as stated by the reptation model. As the leading reptation limiting processes the NSE data have quantitatively confirmed contour length fluctuations destroying the tube confinements from the ends. Also the effect of constraint release has been observed directly on a molecular scale in space and time. It has been experimentally proven that CLF of the matrix chains alone can lead to constraint release.

Compared to the investigations on the dynamics of the synthetic polymers the study of the large scale relaxation dynamics of biopolymers is still in its early stages. We have presented some first experimental data on the component fluctuations of a tetrameric aggregate formed by alcohol dehydrogenase. It became possible to directly measure the $Q$ dependent effective diffusion coefficient which bears information on the detailed rotational diffusion dynamics. This quantity is very sensitive to the actual solution structure and underpins the results from SANS solution structure determination. Furthermore, some additional dynamic effects appear to be related to motions of the outer more flexible parts of each dimer. Finally, the binding of the cofactor NAD reduces the dynamic response in the low $\mathrm{Q}$-flank of $\mathrm{D}_{\text {eff, }}$ an effect possibly related to changes in the configurational stiffness induced by the cofactor.

Aside of the earlier published dynamic data on Taq polymerase [4], where significant domain fluctuations have been observed this is the second experiment of its kind. Future 
experiments will need to resolve the internal dynamics of further proteins and protein complexes trying to resolve the different relaxation modes separately. Such experiments need to be accompanied by computer simulation, in order to enhance the level of interpretation. Furthermore, the experiments need to address proteins where domain motion is functionally important. We hope that in the future such NSE studies will make an important contribution to a better understanding of protein function based not only on the structure but also on the dynamics.

\section{References:}

[1] For a recent review, see, Advances in Polymer Science, ed. D. Richter, M. Monkenbusch, A. Arbe, J. Colmenero (Springer, Berlin, Heidelberg, 2005), Vol. 174.

[2] M. Doi and S.F. Edwards, J. Chem. Soc., Faraday Trans. 274 (1978) 1789; M. Doi and S.F. Edwards, J. Chem. Soc., Faraday Trans. 274 (1978) 1802.

[3] P.G. de Gennes, J. Chem. Phys. 55 (1971) 572.

[4] Z. Bu, R. Biehl, M. Monkenbusch, D. Richter, J.E. Callaway, Proc. Natl. Acad. Sci. U.S.A. 102 (2005) 17646.

[5] Neutron Spin Echo Lecture Notes in Physics, ed. F. Mezei, Springer, Berlin, Heidelberg, 1980, Vol. 128.

[6] A. Wischnewski, M. Monkenbusch, L. Willner, D. Richter, B. Farago, G. Kali, Phys. Rev. Lett. 90 (2003) 058302.

[7] H. Montes, M. Monkenbusch, L. Willner, S. Rathgeber, L.J. Fetters, D. Richter, J. Chem. Phys. 110 (1999) 10188.

[8] P. Schleger, B. Farago, C. Lartigue, A. Kollmar, D. Richter, Phys. Rev. Lett. 81 (1998) 124.

[9] T.C.B. McLeish, Adv. Phys. 51 (2002) 1.

[10] A. Wischnewski, M. Monkenbusch, L. Willner, D. Richter, A.E. Likhtmann, T.C.B. McLeish, B. Farago, Phys. Rev. Lett. 88 (2002) 058301.

[11] M. Zamponi, M. Monkenbusch, L. Willner, A. Wischnewski, B. Farago, D. Richter, Europhys. Lett. 72 (2005) 1039.

[12] M. Zamponi, A. Wischnewski, M. Monkenbusch, L. Willner, D. Richter, A.E. Likhtmann, G. Kali, B. Farago, Phys. Rev. Lett. 96 (2006) 238302.

[13] R. Biehl, M. Monkenbusch, D. Richter, B. Hoffmann, R. Merkel, to be published.

[14] M. Doi and S.F. Edwards, The Theory of Polymer Dynamics, Clarendon, Oxford, 1986.

[15] P.G. de Gennes, J. Phys. (Paris) 42 (1981) 735.

[16] A.E. Likhtmann and T.C.B. McLeish, Macromolecules 35 (2002) 6332.

[17] N. Clark and T.C.B. McLeish, Macromolecules 26 (1993) 5264.

[18] F. Schotte, M. Lim, T.A. Jackson, A.V. Smirnov, J. Soman, J.S. Olson, G.N. Jr Philips, M. Wulff, P.A. Aninfrud, Science 300 (2003) 1944.

[19] D.I. Svergun, Biophys. J. 76 (1999) 2879.

[20] N. Brown, Dynamic Light Scattering (Monographs on the Physics and Chemistry of Materials), Oxford Science Publications, Oxford, Vol. 49, 1993.

[21] J. Garcia de la Torre, M. L. Huertas, B. Carrasco, Biophys. J. 78 (2000) 719. 
